\title{
Comparative study of synthesis and structural by using different precursors of copper oxide nanoparticles and their application in the adsorption capacity
}

\author{
Bahga-Saleh $^{1}$, Samreen Fatema ${ }^{1}$, Mazhar Farooqui ${ }^{1}$, Shaikh Yusuf ${ }^{2}$ \\ ${ }^{1}$ Maulana Azad College of Arts, Science and Commerce, Aurangabad (MS), India \\ ${ }^{2}$ Shivaji College, Kannad (MS), India \\ yshaikh@gmail.com
}

DOI 10.17586/2220-8054-2021-12-2-188-198

Copper oxide nanoparticles were prepared by five sol-gel methods. The comparative study was made by the characterization such as FTIR, SEMEDX, XRD. Out of these protocols, method five is more suitable and gives more accurate and appropriate results. From the data of EDX it is also found that method five gives higher yields of copper oxide nanoparticles. The efficiency of these nanoparticles as low cost adsorbent for the removal of methylene blue (MB) capacity from aqueous solutions was investigated. The effect of different parameters such as amount of adsorbent, concentration of dye, contact time, $\mathrm{pH}$, temperature were studied the adsorption capacity and adsorption behavior of nanoparticles under various conditions. The experimental isotherm data has been studied. The kinetic study of the adsorption obeys Pseudo-first order model. The thermodynamic parameters namely Gibbs free energy, enthalpy, and entropy have revealed that the adsorption of methylene blue on the nanoparticles are feasible, spontaneous and exothermic.

Keywords: nanoparticals, sol-gel method, copper oxide, adsorption, FTIR, SEM-EDX, XRD.

Received: 25 November 2020

Revised: 19 January 2021

\section{Introduction}

Nanotechnology is a rare science that deals with materials in a very small size between $(1-100 \mathrm{~nm})$ with various crystal shapes such as spherical nanoparticles, flower like, nano rods, nano ribbons, nano platelets [1,2]. The synthesis of metal nanoparticles, has been an area of interest recently because of their unusual structural, electrical, optical and magnetic properties. These specific properties of nanoparticles can be adjusted during nanoparticles growth, as these properties are usually dependent upon particle size and surface area [3]. Generally there are numerous metal oxides available in nature but some of the metal oxides are most useful in conjunction with their applications in day to day life in science and technology. In the periodic table transition metals are high in number and have variety of applications in different fields [4]. Copper $(\mathrm{Cu})$ nanoparticles have attracted considerable attention due to their properties among various metal nanoparticles. In recent years, copper oxide has increasingly attracted fundamental as well as practical interests [5]. Cupric oxide $(\mathrm{CuO})$ has a monoclinic crystal structure and a p-type semiconductor with band gap of $1.2-1.9 \mathrm{eV}[6,7]$. It has gained the most interest because of its wide applications, such as in solar transformation and electronics, field emissions, magnetic storage devices, lithium ion batteries, gas sensing, drug delivery, magnetic resonance imaging, and many more. The CuO-NPs also have an application in heat transfer, photoconductive, photothermal and photovoltaic applications. These nanoparticles display superior catalytic activity, with very good selectivity. Moreover they have excellent antimicrobial activity against various bacterial strains. It is used in the fields like catalysis, organic catalysis, photo catalysis, superconductors and ceramics as a kind of important inorganic materials [8-12]. They are used in nano-fluids, where dispersed nanoparticles are used for increase fluid thermal conductivity [13]; athey are also effectively used in anti-fouling paint, wood preservatives, sterile surface coatings, water filters or in textiles and bandages. On the other hand, the biocidal activity of CuO-NPs could be unintentionally harmful to the environment and human health [14]. A low cost production and excellent stability at room temperature, non-toxic nature of the copper oxide material makes it an attractive option for these applications; in addition, strong hole mobility, large minority carrier diffusion length, and a direct energy gap are suitable qualities for efficient absorption. For these applications, $\mathrm{CuO}$ is technologically interesting due to its chemical and physical properties, which depend on its composition, structure, phase, shape, size, and size distribution [15]. There is wide variety of preparation methods for the synthesis of $\mathrm{CuO}$ nanoparticles. Among of these methods our method for the synthesis of $\mathrm{CuO}$ nanoparticles belongs to the chemical method, which is sol-gel method [16]. The sol-gel process involves the formation of a colloidal suspension (sol) and gelatin of the sol to form a network in a continuous liquid phase (gel) [17]. It is one of the most important wet chemical methods used for the preparation of metal oxide nanoparticles [18] and it is widely used due to its diverse advantages. Among the advantages of sol-gel method is the fact that almost every metal oxide structure could be examined and no special apparatus or equipment is required. 
This method works well for the synthesis of high purity complex metal oxides [19]. Several synthetic dyes, including cationic (basic dyes), anionic dyes (direct, acid and reactive dyes), and nonionic dyes (disperse dyes and vat dyes) are widely used in textile, leather and paper dyeing [20]. Some dyes or their metabolites are either toxic or mutagenic and carcinogenic, and thus exert unfavorable effects on the environment and human organs such as the skin and the lungs [21]. There are several methods for removing dyes, which can be categorized into physical, chemical, and biological methods. In general, physical methods, which include adsorption, ion exchange, and membrane filtration are effective for removing reactive dyes without producing undesirable by-products [22, 23]. Great attention has recently been focused on the benefits from processes involving nanoparticles. Nano-sized metal oxides have proven to be effective materials as adsorbents due to their high surface reactivity, adsorption capacity and destructive sorbent compared to their commercial analogues, and their simple synthesis from abundant natural minerals [24]. Adsorption is one of the most studied techniques for the effective removal of dyes primarily due to its simplicity, cost effectiveness and high performance. The effect of various variables such as contact time, $\mathrm{pH}$, adsorbent dosage, effect of volume of dye solution $[25,26]$. Methylene blue $(\mathrm{MB})$ is an essential dye that is used in many industries for coloring and dying purposes and disinfect in pesticides, pharmaceuticals, dye stuffs and varnishes and is commonly adopted as a representative organic pollutant to test the adsorption performance for the removing organic pollutants from waste water $[27,28]$.<smiles>CN(C)c1ccc2nc3ccc(N(C)C)cc3[s+]c2c1</smiles>

FIG. 1. Chemical structure of methylene blue dye

\section{Materials and methods}

In this study, $\mathrm{CuO}$ is prepared by sol-gel method by five different ways. All five nanoparticles were characterized by FT-IR, SEM-EDX, XRD and subjected for the adsorption on Methylene blue by different parameters i.e. change in time, change in concentration of dye, change in weight of adsorbent, change in $\mathrm{pH}$. The variation is presented by graph. For the present work double distilled water is used, copper chloride, sodium hydroxide, methanol, absolute ethanol, oxalic acid, glacial acetic acid, and methylene blue were of AR grade from SD fine chemical Ltd India. Contract analytical services of various labs were taken for SEM, EDX, XRD etc. Five methods were used for the preparation of nanoparticles. These are as follows.

Method 1 (M1): copper oxide NPs prepared by dissolving $2 \mathrm{~g}$ of copper chloride in distilled water. In a separate flask $\mathrm{HCl}\left(36.5 \% \mathrm{HCl}\right.$ in distilled water) and absolute ethanol were refluxed at $70{ }^{\circ} \mathrm{C}$. The $\mathrm{pH}$ was maintained at 3 . The copper solution was added dropwise until the solution gave a black precipitate. The precipitate was centrifuged and kept in muffle furnace at $5000{ }^{\circ} \mathrm{C}$ for two hrs [30].

Method 2 (M2): A solution of $6 \mathrm{gm}$ of oxalic acid in $200 \mathrm{ml}$ of methanol is prepared $0.2 \mathrm{gm}$ of copper chloride is dissolved in about $200 \mathrm{ml}$ of methanol at $35{ }^{\circ} \mathrm{C}$ under constant stirring for $30 \mathrm{~min}$ to obtain a clear solution. The oxalic acid solution was then added to warm solution to yield a thick gel. That solution was subsequently dried in a hot air oven at $80{ }^{\circ} \mathrm{C}$ for $20 \mathrm{hrs}$ to produce copper oxide powder. The powder was subjected to pyrolysis in a muffle furnace at $500{ }^{\circ} \mathrm{C}$ for $2 \mathrm{hrs}$ to give black powder [31].

Method 3 (M3): In the third method of synthesis of copper nanoparticles, $9 \mathrm{gm}$ of copper chloride and $5.4 \mathrm{gm}$ of sodium hydroxide pellet were dissolved in $100 \mathrm{ml}$ methanol separately. A methanolic solution of copper chloride was magnetically stirred. Dropwise addition of sodium hydroxide solution was carried out with continuous stirring at room temperature. The color of the solution was turned to black. The black precipitate was then centrifuged and washed with deionized water and calcined at $450{ }^{\circ} \mathrm{C}$ for $2 \mathrm{hrs}[32]$.

Method 4 (M4): copper nanoparticles were prepared by using 0.2 gm copper chloride was dissolved in $100 \mathrm{ml}$ of double distilled water. This solution was continuously stirred. To this stirred solution $1 \mathrm{ml}$ of glacial acetic acid was added. The solution was heated at $100{ }^{\circ} \mathrm{C}$ for $30 \mathrm{~min}$. Dropwise addition of sodium hydroxide $(8 \mathrm{M})$ was carried out with continuous stirring until the color of solution turned to black from green. The precipitate was centrifuged and washed several time with deionized water and kept in muffle furnace at $450{ }^{\circ} \mathrm{C}$ for $2 \mathrm{hrs}$ [33]. 
Method 5 (M5): $0.2 \mathrm{M}$ of copper chloride was prepared for $\mathrm{CuO}$ nanoparticles. $\mathrm{KOH}$ solution was prepared in benzene and hexane mixture (in 1:1 ratio). This mixture was added to copper chloride solution with continuous stirring. The solution was stirred and refluxed for $2 \mathrm{hrs}$. The precipitate was centrifuged and dried in muffle furnace at $450{ }^{\circ} \mathrm{C}$ for $2 \mathrm{hrs}$ [34].

The nanoparticles so formed were subjected to SEM, EDX and XRD analysis.

\subsection{Adsorption}

The adsorption study was carried out using batch adsorption method. The effect of initial concentration of dye, $\mathrm{pH}$, dosage form, contacts time,etc., was carried out as described in earlier work. The nanoparticles were washed several times with distilled water until it was free from $\mathrm{NaCl}$.

A model anionic dye was selected in this study as because of its complex chemical structure. Solubility in aqueous solution and it is persistence, once it is discharged in to natural environment. The concentration of MB was determined by using UV-visible spectrophotometer (S2-159) at $\lambda_{\max }=660 \mathrm{~nm}$. The adsorption experiments were carried out in stirred batch mode. For experiment, $10 \mathrm{ml}$ of MR, dye solution of specified concentration was continuously stirred unit $0.1 \mathrm{gm}$ of powder at room temperature [35].

\section{Result and discussion}

\subsection{Fourier transform infrared spectroscopy (FTIR) analysis}

The FTIR spectrum of $\mathrm{CuO}$ within the $400-4000 \mathrm{~cm}^{-1}$ range is shown in Fig. 2. FTIR spectra provide information about the nature of the copper oxide nanoparticles are essential for verifying the purity of the oxide. The band at $447.404 \mathrm{~cm}^{-1}$ was ascribed to $\mathrm{CuO}$ vibration, whereas that at $624.823 \mathrm{~cm}^{-1}$ can be assigned to the $\mathrm{Cu}-\mathrm{O}$ stretching [36]. The bandat $578.54 \mathrm{~cm}^{-1}$ indicated the formation of the CuO-NPs [32]. The vibration frequencies at $516.829 \mathrm{~cm}^{-1}$ in the FTIR spectra of $\mathrm{CuO}$ nanoparticles have been assigned to $\mathrm{Cu}-\mathrm{O}$ stretching [37]. A wide absorption band at around $3467.38 \mathrm{~cm}^{-1}$ is observed which occurs due to the absorption of moisture by the nanoparticles. The metal-oxygen bond is observed at $1133.94,1542.77$ and $1654.62 \mathrm{~cm}^{-1}$, indicating the formation of $\mathrm{CuO}$ from Copper Chloride [38].

\subsection{Scanning electron microscopy (SEM-EDX)}

The surface morphologies of the synthesized copper oxide nanoparticles have been examined through scanning electron microscopy. The morphologies of $\mathrm{CuO}$ nanoparticle prepared with different precursors via the sol-gel method. Fig. 3 show the SEM images copper oxide show irregular morphology with different shapes and sizes 58.7, 83.3, 72.4, 46.1 and $83.9 \mathrm{~nm}$. The effect of reaction time plays a significant role in the morphology of nanoparticles.

Figure 4 shows the EDX analysis of CuO-NPs annealed at $450{ }^{\circ} \mathrm{C}$. The EDX analysis was carried out $\mathrm{CuO}$ NPs at $20 \mathrm{keV}$. Results revealed the presence of copper $(\mathrm{Cu})$ and oxygen $(\mathrm{O})$ elements in $\mathrm{CuO}$ in nanoparticles and other peaks are also obtained in EDAX which may be due to the chemicals which were added during processing of nanoparticles. Copper nanoparticles synthesized by five procedures in which M5 gives more amounts of copper nanoparticles is $42.22 \pm 0.82$, while M3 gives least amount of nanoparticles that is $20.48 \pm 0.10$. Table 1 shows the percentage of all elements present in the nano powder.

TABLE 1. Percentage of elements present in the sample from EDX

\begin{tabular}{|c|c|c|c|c|c|}
\hline Element & M1 & M2 & M3 & M4 & M5 \\
\hline $\mathrm{C}$ & $19.18 \pm 0.49$ & $13.25 \pm 0.20$ & $9.84 \pm 0.24$ & $19.21 \pm 0.92$ & $43.22 \pm 1.10$ \\
\hline $\mathrm{O}$ & $33.67 \pm 0.30$ & $33.56 \pm 0.20$ & $22.83 \pm 0.24$ & $29.01 \pm 0.92$ & $37.87 \pm 0.50$ \\
\hline $\mathrm{Na}$ & $1.22 \pm 0.07$ & $\ldots$ & $15.43 \pm 0.17$ & $1.10 \pm 0.48$ & $\ldots$ \\
\hline $\mathrm{Cl}$ & $3.33 \pm 0.05$ & $0.52 \pm 0.01$ & $10.48 \pm 0.10$ & $\ldots$ & $1.21 \pm 0.09$ \\
\hline $\mathrm{Cu}$ & $31.54 \pm 0.15$ & $28.87 \pm 0.07$ & $20.48 \pm 0.10$ & $20.68 \pm 0.39$ & $42.22 \pm 0.82$ \\
\hline
\end{tabular}




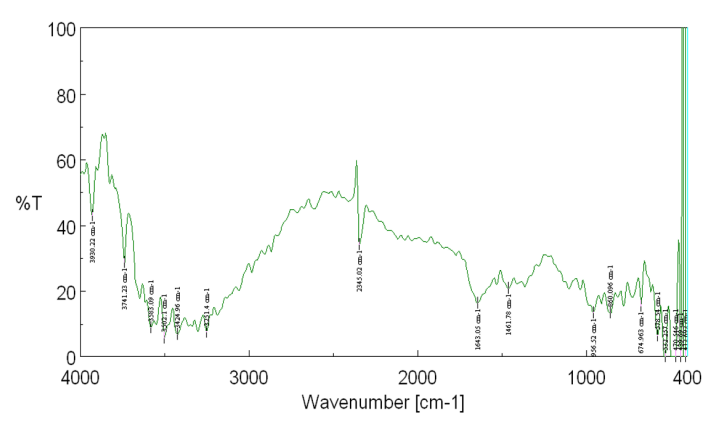

(a)

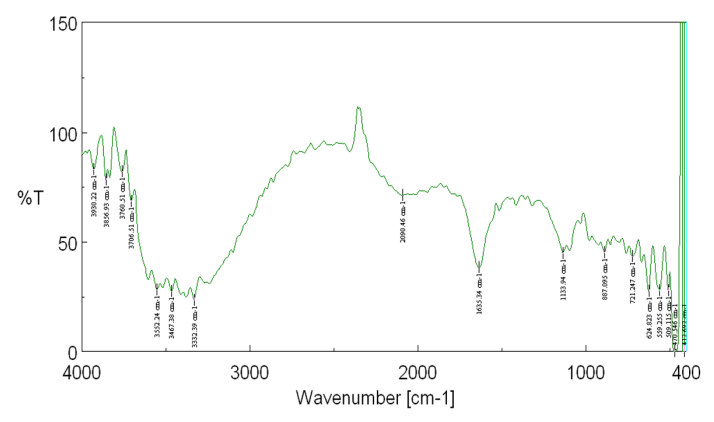

(c)

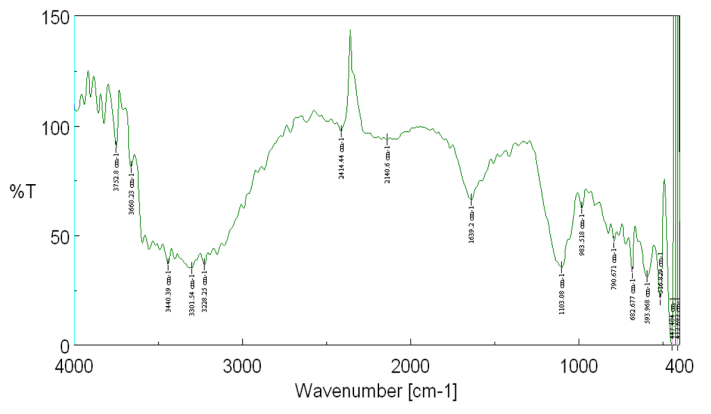

(b)

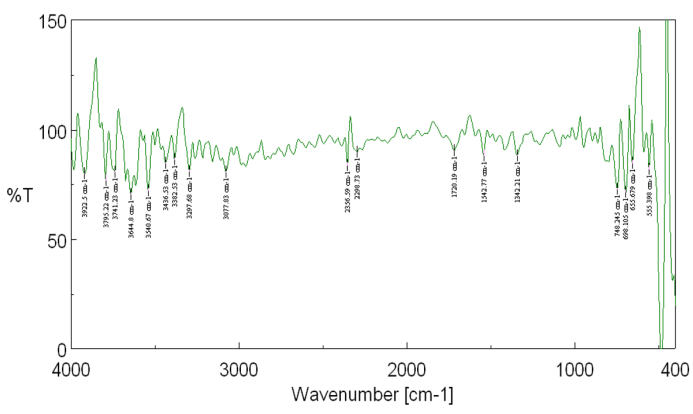

(d)

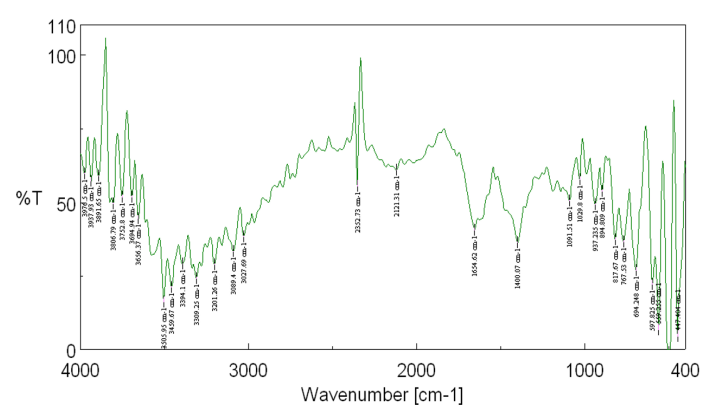

(e)

FIG. 2. FTIR of CnO Nanoparticle: Method 1 (a); Method 2 (b); Method 3 (c); Method 4 (d); Method 5 (e)

\section{3. $\mathrm{X}$-ray diffraction analysis (XRD)}

The XRD patterns of as-synthesized and calcined $\mathrm{CuO}$ nanoparticles using different precursors are shown in Fig. 5. XRD peaks confirm that the formation of $\mathrm{CuO}$ from each precursor was in the monoclinic phase. The figure shows sharp peaks of $\mathrm{CuO}$, which indicate the crystallinity in nature. The XRD pattern of the powder sample.

M1 was recorded in the fraction angle range from 5 to $40^{\circ}$. The characteristic peaks located at $2 \theta=16.53,32.03$, and $35.85^{\circ}$ are assigned to (101), (202) and (210). The highest peak is at angle $32.03^{\circ}$ at (202) plane with 3723.25 intensity account.

$\mathrm{M} 2$ was recorded in the fraction angle range $5^{\circ}$ to $20^{\circ}$. The characteristic peaks located at $2 \theta=7.61^{\circ}, 9.82^{\circ}$ and $15.10 \mathrm{o}$ are assigned to (100), (110) and (200). The highest peak is at angle $7.61^{\circ}$ at (100) plane with 3089.19 intensity.

M3 was recorded in the fraction angle range from 5 to $75^{\circ}$. The characteristic peaks located at $2 \theta=27.36,31.69$, 35. 53, 38.72, 45.42, 56.45 and $66.23^{\circ}$ are assigned to (221), (222), (400), (330), (430), (610) and (632). The highest peak is at angle $31.691^{\circ}$ at (222) plane with 50880.95 intensity.

M4 was recorded in the fraction angle range from 5 to $50^{\circ}$. The characteristic peaks located at $2 \theta=11.53,17.03$, $19.14,22.62,26.98,29.81,31.78,32.61,33.74,34.53,35.62,36.66,37.99,38.81,39.94,41.56,43.41$ and $45.52^{\circ}$ are assigned to (111), (220), (221), (320), (330), (332), (430), (431), (333), (432), (521), (441), (531), (442), (532), (541), (542) and (632). The highest peak is at angle $29.81^{\circ}$ at (332) plane with 24594.34 intensity. 


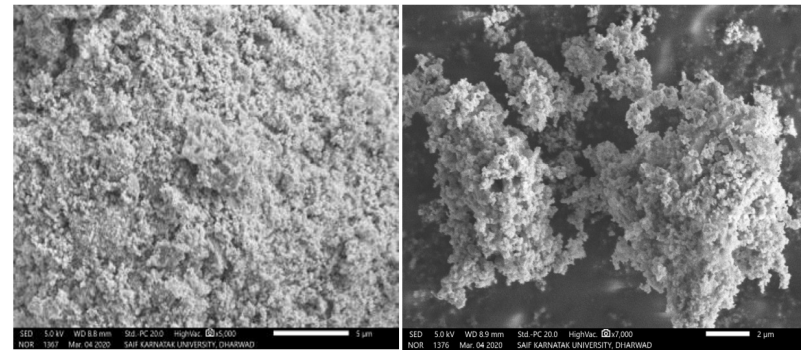

(a)

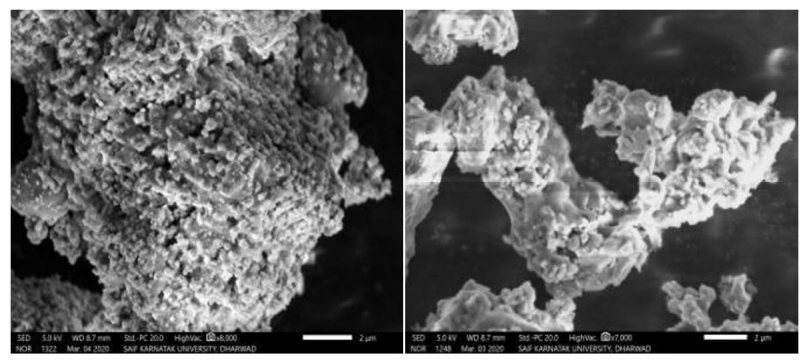

(c)

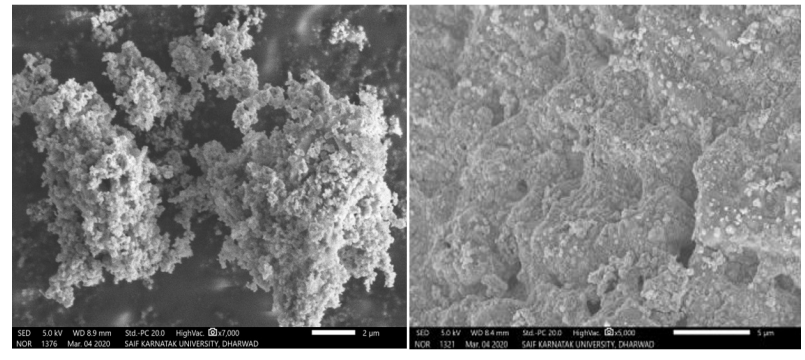

(b)

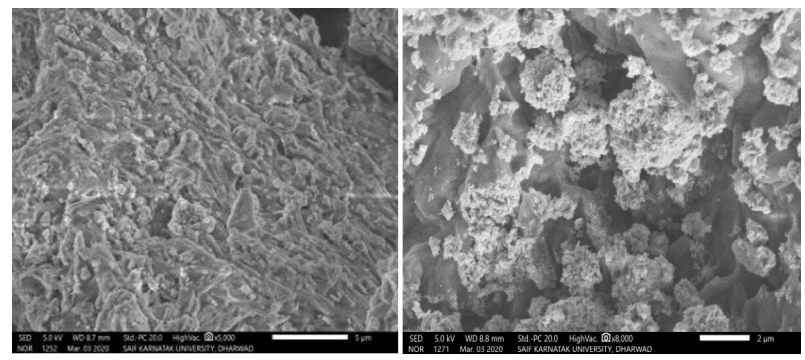

(d)

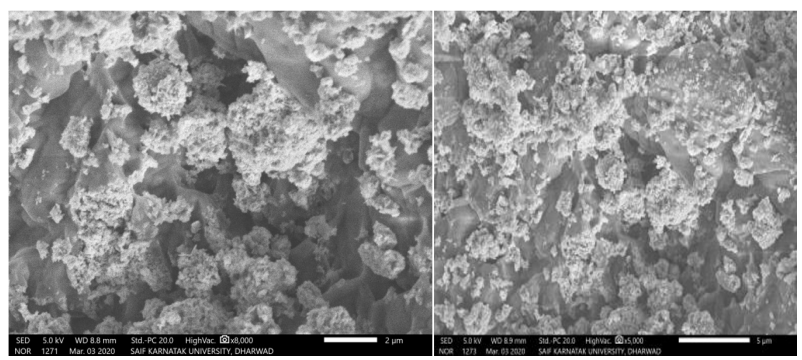

(e)

FIG. 3. SEM images of CuO: Method 1 (a); Method 2 (b); Method 3 (c); Method 4 (d); Method 5 (e)

M5 was recorded in the fraction angle range from 5 to $75^{\circ}$. The characteristic peaks located at $2 \theta=27.41,31.73$, $35.61,38.84,45.47,56.49$ and $66.23^{\circ}$ are assigned to (321), (330), (332), (333), (442), (552) and (660). The highest peak is at angle $31.73^{\circ}$ at (330) plane with 105773.40 intensity.

The average Crystalline sizes respectively 19.9, 27.9, 28.8, 26.9 and $33.6 \mathrm{~nm}$ which calculated from the DebyeScherrer equation [39].

$$
D=\frac{K \lambda}{\beta \cos \theta},
$$

where $D$ is crystallite size $(\mathrm{nm}), K$ - constant $(K=0.9), \lambda$ is X-ray wavelength $(\lambda=0.15406 \mathrm{~nm}), \beta$ is Full-Width Half Maximum (FWHM) of the peak in XRD pattern, $\theta$ is peak obtained angle.

The IR and XRD confirms the confirmation of $\mathrm{CuO}$ nanoparticles. These nanoparticles are used for the evaluation of their adsorption capacities. The SEM images proves that the surface of particles is rough and can adsorb the dye. For present study, Methylene blue (MB) is used as adsorbate.

\subsection{Adsorption study}

In adsorption studies, effect of contact time plays vital role irrespective of other experimental parameters effecting adsorption kinetics. The sample of dye was taken in separate flasks and adsorption studies were carried out at different contact time from 5 to $25 \mathrm{~min}$. The results are given in Fig. 6. 


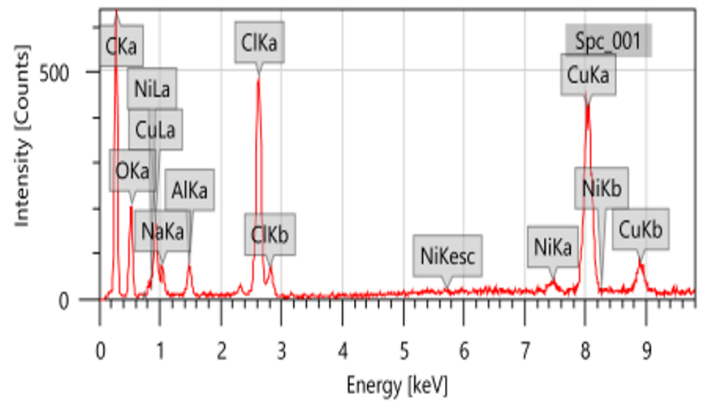

(a)

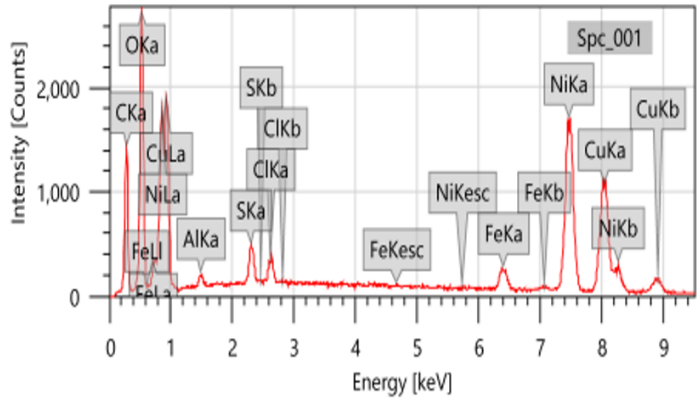

(b)

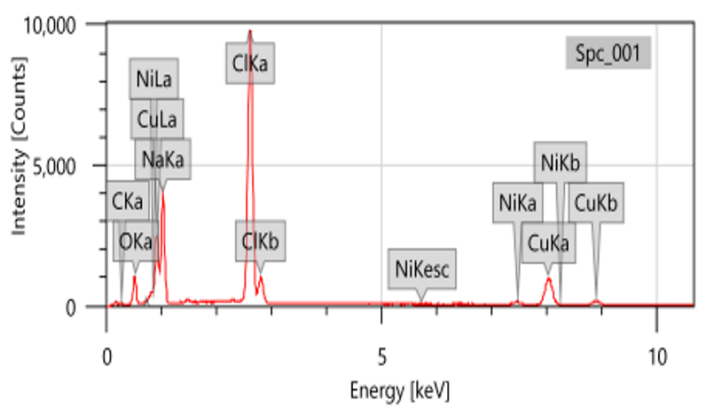

(c)

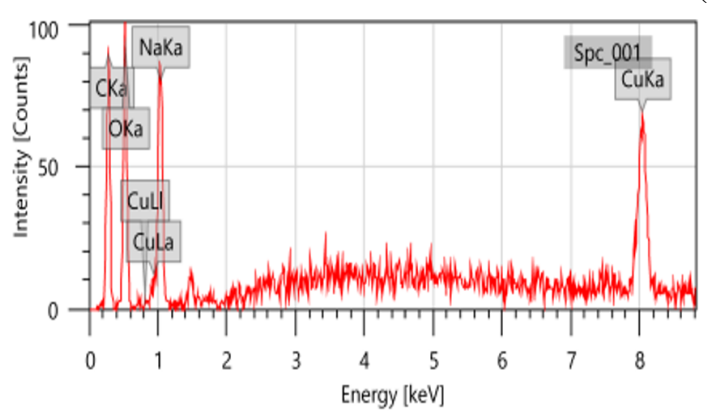

(d)

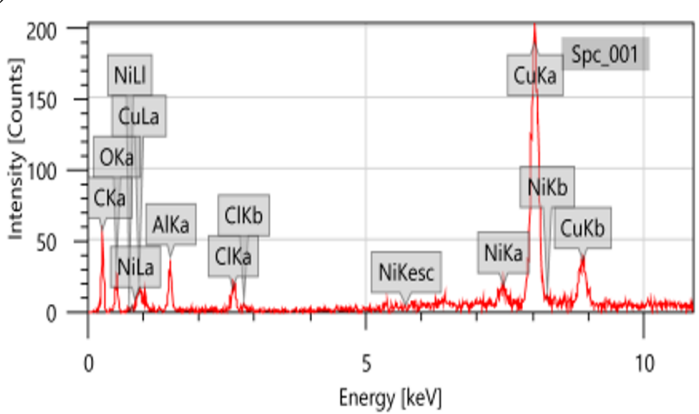

(e)

FIG. 4. FTIR of CnO Nanoparticle: Method 1 (a); Method 2 (b); Method 3 (c); Method 4 (d); Method 5 (e)

\subsection{Adsorption isotherm}

Kinetic isotherm: In the present work, Pseudo first order and Pseudo second order kinetic models can be expressed in a linear form as the following equations. Calculated results for pseudo-first order and second order kinetic are shown in Table 2:

$$
\begin{gathered}
\log \left(q_{e}-q_{t}\right)=\log q_{e} \frac{K_{1} t}{2.303}, \\
\frac{t}{q_{t}}=\frac{1}{K_{2} q_{e}^{2}}+\frac{t}{q_{e}},
\end{gathered}
$$

where $q_{e}$ and $q_{t}$ are the amount of MB adsorbed at equilibrium and different times (min) respectively $\mathrm{K} 1$ represents the first order constant $\left(\mathrm{min}^{-1}\right)$ [40-42].

The equilibrium adsorption data have been analyzed using well known Langmuir and Freundlich model. A mathematical expression of the Langmuir isotherm is given by the following equation:

$$
\frac{C_{e}}{Q_{e}}=\frac{1}{Q^{0}}+\frac{C_{e}}{Q^{0}}
$$

where $C_{e}$ is the equilibrium concentration of the $\mathrm{MB}, Q_{e}$ is $\mathrm{MB}$ amount adsorbed at equilibrium $q_{m}$ and $K_{L}$ are Langmuir constants related to the adsorption capacity and adsorption energy, which can be calculated from linear regression of $C_{e} / Q_{e} \mathrm{~V} / \mathrm{s} C_{e}$. 


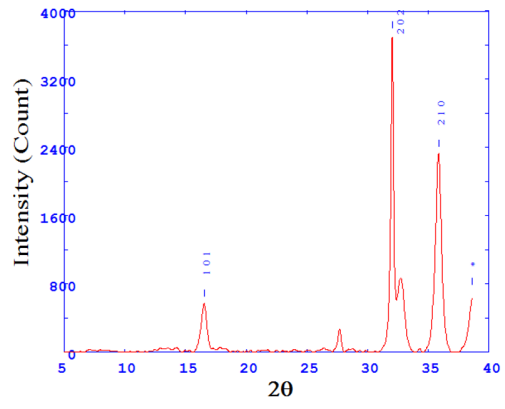

(a)

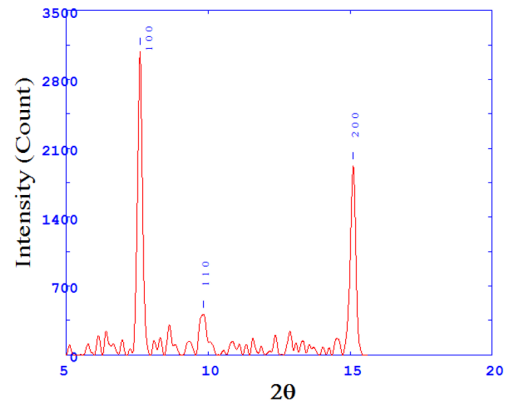

(b)

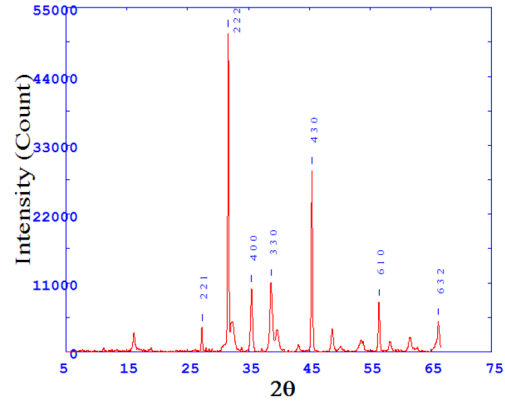

(c)

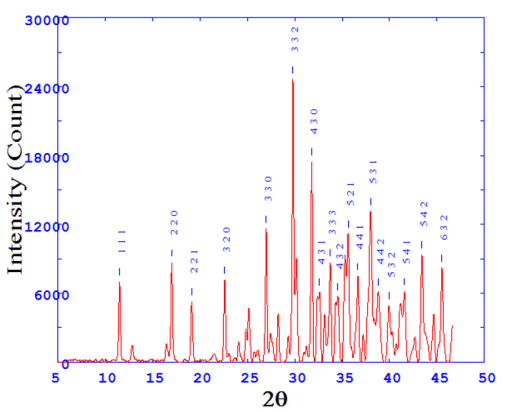

(d)

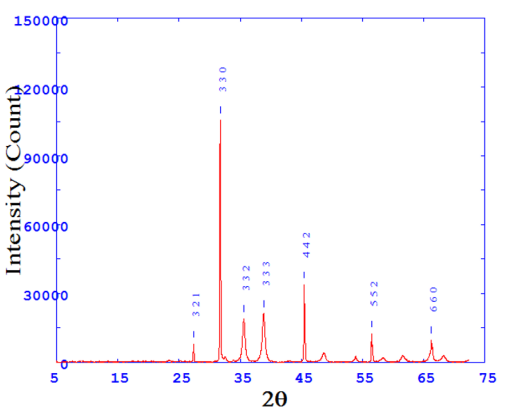

(e)

FIG. 5. XRD spectra of CuO: Method 1 (a); Method 2 (b); Method 3 (c); Method 4 (d); Method 5 (e)

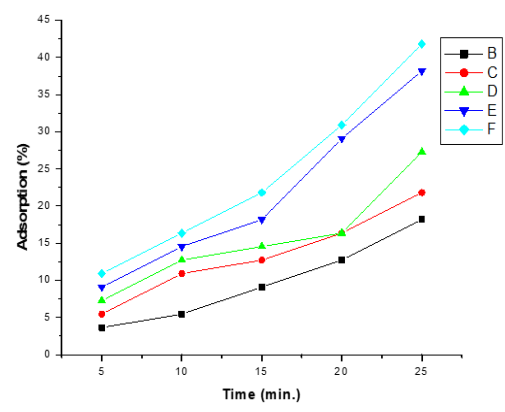

(a)

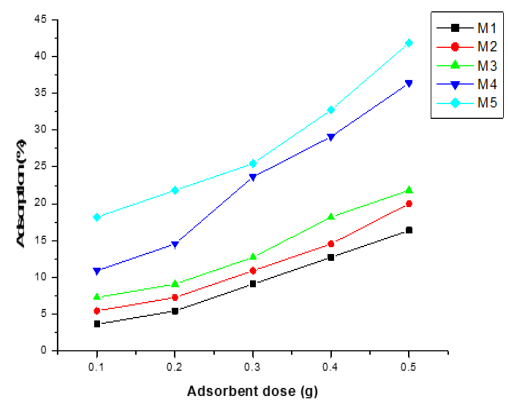

(b)

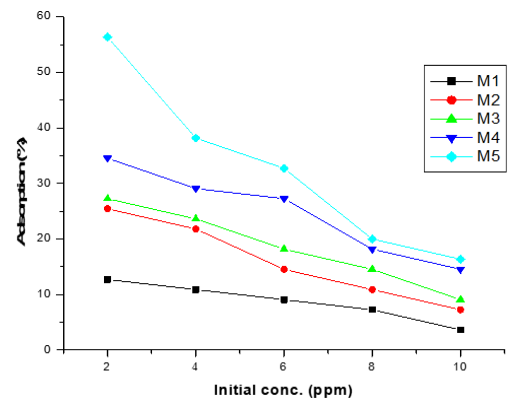

(c)

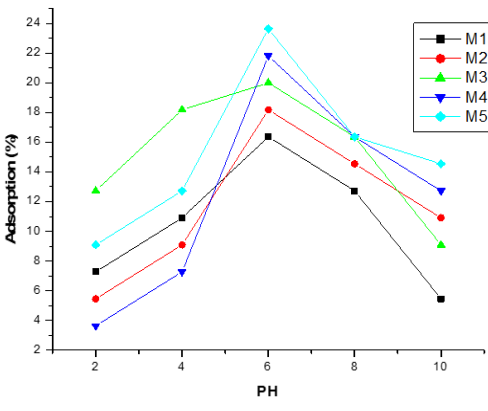

(d)

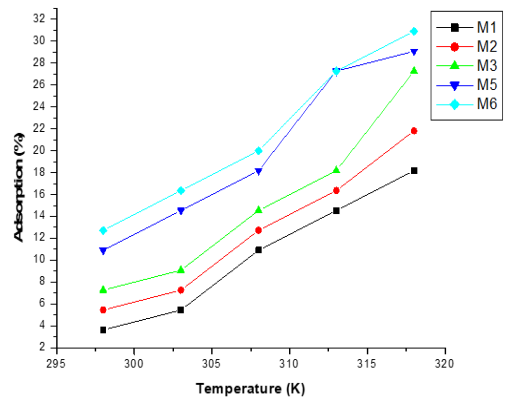

(e)

FIG. 6. Variation in time (a); Variation in adsorbent dose (b); Variation in concentration (c); Variation of $\mathrm{pH}(\mathrm{d})$; Variation in temperature (e) 
TABlE 2. Comparison of the experiments and the kinetic model of $\mathrm{MB}$ dye on $\mathrm{MnO}_{2}$ adsorbent

\begin{tabular}{|c|c|c|c|c|c|c|c|}
\hline & \multirow{2}{*}{$\begin{array}{l}\text { Conc. } \\
\text { of MR } \\
(\mathrm{mg} / \mathrm{L})\end{array}$} & \multicolumn{3}{|c|}{ Pseudo-first order } & \multicolumn{3}{|c|}{ Second order } \\
\hline & & $\begin{array}{c}K_{1} \\
\left(\min ^{-1}\right)\end{array}$ & $\begin{array}{c}q_{e} \\
(\mathrm{mg} / \mathrm{gm})\end{array}$ & $R_{2}$ & $\begin{array}{c}K_{2} \\
(\mathrm{gm} / \mathrm{mg} \cdot \min )\end{array}$ & $\begin{array}{c}q_{e} \\
(\mathrm{mg} / \mathrm{gm})\end{array}$ & $R_{2}$ \\
\hline M1 & 10 & 0.08731 & 8192.756 & 0.96054 & $1.71 \times 10^{-7}$ & 19091.26 & 0.045309 \\
\hline M2 & 10 & 0.11852 & 8732.930 & 0.88619 & $3.98 \times 10^{-7}$ & 16366.61 & 0.095704 \\
\hline M3 & 10 & 0.10684 & 8425.972 & 0.86020 & $2.08 \times 10^{-6}$ & -10687.00 & 0.164877 \\
\hline M4 & 10 & 0.22498 & 8300.419 & 0.93805 & $2.84 \times 10^{-7}$ & 23866.35 & 0.113529 \\
\hline M5 & 10 & 2.24596 & 8724.690 & 0.94341 & $7.84 \times 10^{-7}$ & 15878.06 & 0.329866 \\
\hline
\end{tabular}

A dimensionless constant separation factor $R_{L}$ is used to express the essential characteristic of the Langmuir isotherm, which is given by:

$$
R_{L}=\frac{1}{1-b C_{0}},
$$

where $C_{0}(\mathrm{mg} / \mathrm{L})$ is the initial in liquid phase. The values of $R_{L}$ indicates the adsorption isotherm model of characteristic as follows: $R_{L}>1$ (unfavorable), $R_{L}=1$ (Linear), $0<R_{L}<1$ (favorable) and $R_{L}=0$ (irreversible). Results are shown in Table 3.

TABLE 3. Adsorption constant

\begin{tabular}{|c|c|c|c|c|c|c|c|c|}
\hline & $\begin{array}{c}\text { Conc. } \\
\text { of MR } \\
(\mathrm{mg} / \mathrm{L})\end{array}$ & $\begin{array}{c}Q_{0} \\
(\mathrm{mg} / \mathrm{g})\end{array}$ & $\begin{array}{c}b \cdot 10^{-5} \\
(\mathrm{~L} / \mathrm{g})\end{array}$ & $R_{L}$ & $R_{2}$ & $n$ & \multicolumn{3}{c|}{ Freundlich constant } \\
\cline { 3 - 8 } & & $-43 \mathrm{mg} / \mathrm{g} \cdot(\mathrm{L} / \mathrm{g})) 1 / n$ & $R_{2}$ \\
\hline M1 & 10 & -4376.37 & -0.03510 & -0.39841 & 0.985652 & 0.21211 & 0.000156 & 0.919432 \\
M2 & 10 & -3385.24 & -0.02902 & -0.52583 & 0.988451 & 0.18504 & $4.98 \times 10^{-6}$ & 0.921468 \\
M3 & 10 & -3784.44 & -0.03051 & -0.4875 & 0.987042 & 0.19826 & $2.38 \times 10^{-5}$ & 0.920180 \\
M4 & 10 & -1631.85 & -0.02061 & -0.94293 & 0.992693 & 0.12191 & $1.14 \times 10^{-11}$ & 0.981394 \\
M5 & 10 & -4194.63 & -0.02135 & -0.66017 & 0.984004 & 0.21501 & $7.75 \times 10^{-5}$ & 0.925983 \\
\hline
\end{tabular}

Compared with the Langmuir and Freundlich isotherm accredit the heterogeneity of the surface and consider, the adsorption occurs at sites with different energy of adsorption. This equation can also apply for multilayer adsorption and expressed by the following equation:

$$
\log q_{e}=\log K_{f}+\frac{1}{n \log C_{e}},
$$

where $K_{f}$ and $n$ are the adsorption capacity of the adsorbent and adsorption intensities, respectively. The values of $K_{f}$ and $n$ are calculated from the interception and slope of the plot of $\log q_{e}$ v/s $\log C_{e}$ [43-45].

Thermodynamic parameter: To determine the possibility of spontaneously occurring of the MB adsorption, thermodynamic parameters were calculated. These parameters such as change in standard Gibb's free energy $\left(\Delta G^{0}\right.$, $\mathrm{J} / \mathrm{mol} / \mathrm{K})$, enthalpy $\left(\Delta H^{0}, \mathrm{KJ} / \mathrm{mol}\right)$, and entropy $\left(\Delta S^{0}, \mathrm{~J} / \mathrm{mol} / \mathrm{K}\right)$ were determine from experimental data observed over the temperature range of $303-323 \mathrm{~K}$. These parameters can be calculated as:

$$
\begin{gathered}
K_{e}=\frac{q_{e}}{C_{e}}, \\
\Delta G^{0}=-R T \log K_{e}, \\
\ln K_{e}=\frac{\Delta S^{0}}{R}-\frac{\Delta H^{0}}{R T},
\end{gathered}
$$


where $K_{e}$ is the equilibrium constant defined by $q_{e} / C_{e}\left(q_{e}\right.$ is the equilibrium amount of adsorbate that adsorbed on adsorbent surface $(\mathrm{mg} / \mathrm{L})$ and $C_{e}$ is the equilibrium amount of unadsorbed remains in the solution). $R$ and $T$ are the universal gas constant. The values for $\Delta H^{0}$ and $\Delta S^{0}$ can be calculated using the plot of $K_{d}$ v/s $1 / T$ (Table 4 and Table 5) [46].

TABLE 4. Thermodynamic parameter values

\begin{tabular}{|c|c|c|c|c|c|c|}
\hline $\begin{array}{c}\text { Sr. } \\
\text { No. }\end{array}$ & $\begin{array}{c}\text { Temp. } \\
(\mathrm{K})\end{array}$ & $\Delta G^{0}(\mathrm{M} 1)$ & $\Delta G^{0}(\mathrm{M} 2)$ & $\Delta G^{0}(\mathrm{M} 3)$ & $\Delta G^{0}(\mathrm{M} 4)$ & $\Delta G^{0}(\mathrm{M} 5)$ \\
\hline 1 & 298 & 37943.4089 & 52328.80669 & 33185.6802 & 27394.87892 & 30092.49601 \\
2 & 303 & 38495.7025 & 53108.85743 & 33665.4808 & 27793.94939 & 30544.53897 \\
3 & 308 & 39047.9961 & 53888.90816 & 34145.2813 & 28193.01987 & 30996.58193 \\
4 & 313 & 39600.2896 & 54668.9589 & 34625.0819 & 28592.09035 & 31448.62489 \\
5 & 318 & 40152.5832 & 55449.00963 & 35104.8825 & 28991.16083 & 31900.66785 \\
\hline
\end{tabular}

TABLE 5. Values of entropy and enthaply

\begin{tabular}{|c|c|c|c|}
\hline Sr. No. & Method & $\Delta S^{0}$ & $\Delta H^{0}$ \\
\hline 1 & M1 & -55.22935668 & 21208.91391 \\
2 & M2 & -78.00507357 & 28693.2694 \\
3 & M3 & -47.98005725 & 18647.72288 \\
4 & M4 & -39.90704777 & 15303.04344 \\
5 & M5 & -45.20429608 & 16395.5943 \\
\hline
\end{tabular}

\section{Conclusion}

The present study concludes that all the considered methods are good for the preparation of nanoparticles but fifth method is more appropriate and gives higher yields of copper oxide nanoparticle. The results of FTIR, SEM-EDX and XRD of all five samples reveals the presence of nanoparticles. The synthesized nanoparticles were studied for the removal of dyes from aqueous solutions. It was found that removal of $\mathrm{MB}$ dye from aqueous solutions using these synthesized nanoparticles is both highly efficient and environmentally-friendly. In this respect, the adsorption is followed by first a pseudo-first order model. The thermodynamic parameters such as Gibb's free energy, enthalpy, and entropy have revealed that the adsorption of methylene blue on the nanoparticles is feasible, spontaneous and exothermic.

\section{References}

[1] Saja M.J. Synthesis of CuO Nano structure via sol-gel and Precipitation Chemical Methods. Al-Khwarizmi Engineering Journal, 2016, 12 (4), P. 126-131.

[2] Hadeel K.T., Kadhim A.H., Ahmed A.A. Synthesis of copper oxide nanoparticles via sol-gel method. Int. Journal of Research in Engineering and Innovation, 2017, 1 (4), P. 43-45.

[3] Jeevan J.M., Umesh K.G. Sol-gel Synthesis of Copper, Silver and Nickel Nanoparticles and Comparison of their Antibacterial activity. Int. Journal of Theor. \& Appl. Sciences, 2017, 9 (2), P. 151-156.

[4] Kankanit P., Sineenart S., Wanichaya M., Wisanu P. Synthesis of CuO Nanoparticles by Precipitation Method Using Different Precursors. Energy Procedia, 2013, 3 (4), P. 740-745.

[5] Rejitha S.G., Krishnanb C. Synthesis of cadmium-doped copper oxide nanoparticles: Optical and structural characterizations. Advances in Applied Science Research, 2013, 4 (2), P. 103-109.

[6] Daniel-Umeri R., Osuji F.I., Ezema R.U. Synthesis and Characterization of Copper Oxide Thin Films Using Successive Ionic Layer Adsorption Reaction (SILAR) Method. Chemistry and Materials Research, 2016, 8 (6), P. 68-76. 
[7] Sanjay S., Mahendra K., Arvind A., Sudhanshu K.D. Synthesis and Characterisation of Copper Oxide nanoparticles. IOSR Journal of Applied Physics (IOSR-JAP), 2013, 5 (4), P. 61-65.

[8] Etefagh R., Azhir E., Shahtahmasebi N. Synthesis of $\mathrm{CuO}$ nanoparticles and fabrication of nanostructural layer biosensors for detecting Aspergillus niger fungi. Scientia Iranica Transactions F: Nanotechnology, 2013, 20 (3), P. 1055-1058.

[9] Nithya K., Yuvasree P., et al. Preparation and Characterization of Copper Oxide Nanoparticles. Int. Journal of ChemTech Research, 2014, 6 (3), P. 2220-2222.

[10] TranT. H., Nguyen V.T. Copper Oxide Nanomaterials Prepared by Solution Methods, Some Properties, and Potential Applications. Int. Scholarly Research Notices, 2014, 856592.

[11] Soltanianfard M.J., Firoozadeh A. Synthesis and Characterization of Copper (II)-Oxide Nanoparticles from Two Cu (II) Coordination Polymers. Journal of Sciences, Islamic Republic of Iran, 2016, 27 (2), P. 113-117.

[12] Khashan K.S., Jabir M.S., Abdulameer F.A. Preparation and characterization of copper oxide nanoparticles decorated carbon nanoparticles using laser ablation in liquid. Journal of Physics: Conf. Series, 2018, 1003, 012100.

[13] Manimaran R., Palaniradja K., et al. Preparation and characterization of copper oxide nanofluid for heat transfer applications. Appl. Nanosci., 2014, 4, P. 163-167.

[14] Rejitha S.G., Krishnan C. Synthesis of cadmium-doped copper oxide nanoparticles: Optical and structural characterizations. Advances in Applied Science Research, 2013, 4 (2), P. 103-109.

[15] Diachenko O.V., Opanasuyk A.S., et al. Structural Features of Nanostructured Copper Oxide Thin Films, Synthesized by Spray Pyrolysis Technique. Proc. Nap, 2015, 4 (4), P. 1-4.

[16] Aparna Y., Enkateswara Rao K.V., Srinivasa Subbarao P. Synthesis and Characterization of CuO Nano Particles by Novel Sol-Gel Method. IPCBEE, 2012, 48.

[17] Jagdeep M., Kshirsagar S., Ramakant S., Prakash S.A. Preparation and characterization of copper oxide nanoparticles critical heat flux. Thermal science, 2017, 21 (1A), P. 233-242.

[18] Arindam G., Navnita K., Ayon B. Investigations on structural and optical properties of Cu doped ZnO. Journal of NanoScience and NanoTechnology, 2014, 2 (4), P. 485-489.

[19] Catherine W.C.L. Synthesis and Characterization of Manganese Dioxide Nanoparticles and Thin Films. A dissertation submitted in partial fulfilment of the requirement for the degree of Bachelor of Science, University Malaysia Sarawak, 2007.

[20] Radwan N.R.E., Hagar M., Chaieb K. Adsorption of Crystal Violet Dye on Modified Bentonites. Asian Journal of Chemistry, 2016, 28 (8), P. 1643-1647.

[21] Ameeth B.I., Nagalakshmi R., Shanthi T. Removal of congo red and magenta dyes from industrial waste water by thorn apple leaf powder. Int. J. Chem. Sci, 2016, 14 (1), P. 57-64.

[22] Subir C., Tapan K.S. Adsorption of Reactive Blue 4 (RB4) onto Rice Husk in Aqueous Solution. Int. Journal of Scientific \& Engineering Research, 2016, 7 (3), P. 7-12.

[23] Masoud S-R. Mohsen I., et al. Removal, preconcentration and determination of methyl red in water samples using silica coated magnetic nanoparticles. Journal of Applied Research in Water and Wastewater, 2014, 1, P. 6-12.

[24] Muhammad N.Z., Qamar D., et al. Effective adsorptive removal of azo dyes over spherical ZnO. JMR\&T journal research and technology, 2019,8 (1), P. 713-725.

[25] Maryam N.A., Niyaz M.M., et al. Adsorption of organic dyes using copper oxide nanoparticles: isotherm and kinetic studies. Journal Desalination and Water Treatment, 2016, 57 (52), P. 25278-25287.

[26] Oannis A., Ahmad H-B, et al. Use of nanoparticles for dye adsorption: Review. Journal of Dispersion Science and Technology, 2018, 39 (6), P. 836-847.

[27] Li L.H., Xiao J., Liu P., Yang G.W. Super adsorption capability from amorphousization of metal oxide nanoparticles for dye removal. Science Reports, 2015, 5, 9028.

[28] Chandrakala M., Anima U. Adsorption Efficiency of Phosphoric Acid Activated Gulmohar (Delonix regia) Fruit Shell Charcoal using Methylene Blue. Int. Journal for Research in Applied Science \& Engineering Technology, 2018, 6 (4), P. $480-484$.

[29] Lotfi M., Lazhar B., et al. Removal of methylene blue from aqueous solutions by adsorption on kaolin: Kinetic and equilibrium studies. Applied clay science, 2018, 153, P. 38-45.

[30] Sanchez E., Lapez T. Effect of the preparation method on the band gap of titania and platinum-titania sol-gel materials. Mat. latt., 1995, 25, P. 271-275.

[31] Parmvir S., Rishab J., et al. Synthesis of Manganese Oxide NPs Using Similar Process and Different Precursors — A Comparative Study. Nanotech R. J., 2015, 8 (3), P. 419-427.

[32] Ismat Z.L., Lutfun N.H., et al. Preparation and Characterization of Copper Oxide NPs Synthesized via Chemical Precipitation Method. Open Access Library Journal, 2015, 2, e1409.

[33] Hadeel K.T., Kadhim A.H., Al-Amiery A.A. Synthesis of copper oxide NPs via sol-gel method. IJREI, 2017,1 (4), P. 43-45.

[34] Sanjay S., Mahendra K., Arvind A., Sudhanshu K.D. Synthesis and Characterisation of Copper Oxide NPs. IOSR-JAP, 2013, 5 (4), P. 61-65.

[35] Rajesh D., Milind U., Farooqui M., Shantilal R. Adsorption study for the Removal of Hazrdous Dye Congo Red by Biowaste Materials as Adsorbents. IJAIEM, 2016, 5 (11).

[36] Anita S.E., Dae J.K. Synthesis and characterization of CuO nanowires by a simple wet chemical method. Ethiraj and Kang Nanoscale Research Letters, 2012, 7, 70

[37] Ensieh S., Nourollah F., Aliakbar D.K. Copper Oxide Nanoparticles Prepared by Solid State Thermal Decomposition: Synthesis and Characterization. Journal of Ultrafine Grained and Nanostructured Materials, 2016, 49 (1), P. 48-50.

[38] Kalita C., Karmakar S. Analysis of Structural and Optical Features of CuO Nanoparticles Synthesized at Different Molarities. Int. Journal of Scientific Research in Physics and Applied Sciences, 2018, 6 (2), P. 30-34.

[39] Deepak P., Shikha S., Pardeep S. Removal of methylene blue by adsorption ontoactivated carbon developed from Ficus carica bast. Arabian Journal of Chemistry, 2017, 10, P. 1445-1451.

[40] Soheila H., Marzieh T., Seyed J.S. Removal of crystal violet from water by magnetically modified activated carbon andnanomagnetic iron oxide. Journal of Environmental Health Science \& Engineering, 2015, P. 2-7. 
[41] Dattatraya J., Bashir A.D., Farooqui M. Husk of gram seeds as a low-cost adsorbent for the removal of methylene blue dye from aqueous solutions. Journal of enviromental science and water resources, 2013, 2 (7), P. 226-232.

[42] Abdo T., Mohd M., Farooqui M., Maqdoom F. Studies on the isotherms, kinetics and thermodynamics of adsorption of crystal violet on low cost materials. Journal of advanced scientific research, 2012, 3 (1), P. 36-44.

[43] Jiancheng S., Renlog L., et al. Adsorption of methylene blue on modified electrolytic manganse residue: Kinetics, isotherm, thermodynamics and mechanism analysis. Journal of Taiwan Inst. of Chem. Eng., 2018, 82, P. 351-359.

[44] Jirekar D.B., Ghumare P., Farooqui M. Kinetics and isotherm studies on crystal violet dye adsorption onto black gram seed husk. Int. Journal of Chemtech research, 2015, 7 (1), P. 427-434.

[45] Jirekar D.B., Farooqui M. Adsorption of congo red dye from aqueous solution using ecofriendly low cost material prepared from Cicerarientinum. Arab journal of physical chemistry, 2015, 2 (1).

[46] Youssef M., Anisa L., et al. Adsorption of methylene blue dye from aqueous solutions onto walnut shell powder: equilibrium and kinetics studies. Surface and interfaces, 2018, 11, P. 74-81.

[47] Sanaz M., Dariush B., Hadi S. Methylene blue removal using modified celery (Apium graveolens) as a low cost biosorbent in batch mode: Kinetic, equilibrium and thermodynamic studies. Journal of molecular structure, 2018, 1173, P. 541-551. 